\title{
Automatic segmentation of ceramic materials with relaxed possibilistic c-means clustering for defect detection
}

\author{
Kwang Baek Kim ${ }^{1}$, Doo Heon Song ${ }^{2}$, Hyun Jun Park ${ }^{3}$ \\ ${ }^{1}$ Division of Computer and Information Engineering, Silla University, Korea \\ ${ }^{2}$ Department of Computer Games, Yong-In SongDam College, Korea \\ ${ }^{3}$ Division of Software Convergence, Cheongju University, Korea
}

\begin{tabular}{l} 
Article Info \\
\hline Article history: \\
Received Jan 10, 2020 \\
Revised Mar 1, 2020 \\
Accepted Mar 17, 2020 \\
\hline Keywords: \\
Ceramic material \\
Defect detection \\
Fuzzy stretching \\
Pixel clustering \\
Possibilistic c-means
\end{tabular}

\begin{abstract}
Automatic inspection system is necessary for reliable quality control if ceramic materials to avoid operator subjectivity and fatigue in visual inspection. Automatic segmentation from material's image is then the most important process to develop such an inspection system. In this paper, we propose a Possibilistic C-Means pixel clustering algorithm with fuzzy stretching to form the defect object in segmentation. In experiment using 50 images containing a certain amount of defects, the proposed method was successful in 49 cases or $98 \%$ of opportunities. That performance is roughly twice better than that of standard K-means clustering in defect object formation.
\end{abstract}

Copyright $(2020$ Institute of Advanced Engineering and Science. All rights reserved.

\section{Corresponding Author:}

Kwang Baek Kim,

Division of Computer and Information Engineering,

Silla University,

Busan 46958, Korea.

Email :gbkim@silla.ac.kr

\section{INTRODUCTION}

Manufacturing of ceramic parts frequently leads to cracks on the surface if the requisite precision and durability are not met with high quality, which can lead to potentially dangerous equipment failure. However, the process of visual inspection often causes subjective decision and relies on the experience of the inspector thus the reliability of such test might be affected and often time-consuming and expensive. The use of appropriate nondestructive evaluation approache is critical to effective process/quality control of the product and reliable performance in service. Ceramic materials that have high thermal resistance, low density, and high degree of hardness are a traditional non-destructive testing application area. However, cracks, spiracles, and other foreign substances that form various defects on the surface have negative influence on its reliability and hardness [1].

Non-destructive visual inspection for texture and/or color abnormalities has applied to various surfaces of objects such as wood, steel, wafer, and ceramics. However any visual inspection of surface structure health monitoring brings the subjectivity problem and the cost-ineffective repetitive process issue thus an automated computer assisted inspection system is highly demanded by industry [2]. Automatic intelligent defect detection systems that can avoid such subjectivity can be classified into two categories with respect to the characteristics of materials. While inspecting materials such as textile, ceramics, plastics, etc should deal with the stochastic variations in scale and other environmental factors in detection of abnormality, and inspecting traditional crack detection on the surface of uniform structures such as bridge, wood, concrete etc. focuses the identification of regions that differ from a uniform background [3]. The automated material inspection system with intelligent image analysis typically consists of processes such 
as image acquisition, image enhancement, image segmentation, feature extraction and classification [4]. However, segmentation step remains a very delicate process since there are many occasions having irregular types of noise and low contrasted images [5]. From different perspective, adaptive edge detecting algorithms with contour analysis [6-8] have been used in defect detection but these techniques are argued due to its sensitivity to the noise and requiring domain specific patterns and statistical support [9]. Some other systems try to combine machine learning methods and image processing techniques to deal with this problem such as fuzzy thresholding technique [8] and Expectation Maximization (EM) algorithm to find appropriate threshold from ultrasound image [10] or use neural network in classification of detected cracks [11-12] but they are sensitive to the brightness contrast of given images.

The main difficulty of developing highly reliable automatic inspection lies to the image segmentation phase where efficient noise removal and enhancement of the image contrast to detect the defects from the background. Thus, previously we focused on the image filtering and binarization method to make the base image having enough contrast from the background in defect detection [13-15].

In this paper, we propose a pixel clustering based automatic segmentation of ceramic materials of various thickness used in welding process. Pixel clustering methods are designed to enhance the brightness contrast and form an object from input image. Automatic detection of an object from low contrast images by pixel clustering have been successful in medical domain with K-means [16-18] and Fuzzy C-means algorithm [19-21] as well as ART2 clustering in ceramic defect detection [2]. While being effective in most cases, such strategies need careful preprocessing for noise removal and image contrast enhancement. Also, ART2 learning was undesirably sensitive to the boundary variable values so that the scalability of such strategy was questioned [15]. To overcome those deficits in pixel clustering procedure, we adopt Possibilistic C-Means (PCM) clustering strategy. PCM has long history of theoretical background [22, 23] and many successful applications in engineering [24, 25] and medical domain [26, 27] and many constraint relaxed models exist [28]. We also relax some part of standard PCM as proposed in [22] to adapt the domain constraints and due to the low intensity contrast between the target area and the background, we need fuzzy stretching [2] to enhance the contrast.

\section{METHOD}

\subsection{Adaptive fuzzy stretching for contrast enhancement}

In preprocessing for the automatic segmentation, we need to enhance the contrast of the image such that the target object has enough discriminative characteristics from the background or non-target object. However, the purpose of this paper is not finding the best preprocessing steps but to test the feasibility of a good pixel clustering algorithm to form the target object, we apply the standard Gaussian filtering and MaxMin binarization with 3 x 3 Sobel mask [29]. Very frequently, any vision based object extraction procedure provides auxiliary intensity stretching algorithm to extend the contrast to differentiate the target object from the background effectively. In this paper, we modify the fuzzy stretching procedure to add adaptability to the environment of input image as following;

The first step is to compute the average brightness value by using this formula:

$$
x_{m}=\sum_{i=0}^{255} x_{i} \frac{1}{M N},
$$

where $M$ and $N$ denote the width and length of the image.

Then the distance between the brighter, the darker area and the average are computed

$$
\begin{aligned}
& d_{\text {max }}=\left|x_{h}-x_{m}\right|, \\
& d_{\text {min }}=\left|x_{m}-x_{l}\right|,
\end{aligned}
$$

where $x_{h}$ and $x_{l}$ is the highest and the lowest intensity pixel value, in respectively.

The brightness value us adjusted using the following rule:

$$
\begin{gathered}
\text { If }\left(x_{m}>128\right) a d=255-x_{m} \\
\text { Else if }\left(x_{m} \leq d_{\text {min }}\right) a d=d_{\text {min }} \\
\text { Else if }\left(x_{m} \leq d_{\max }\right) a \mathrm{~d}=d_{\max } \\
\text { Else } a d=x_{m} \\
I_{\max }=x_{m}+a d \\
I_{\min }=x_{m}-a d
\end{gathered}
$$


where $I_{\max }$ and $I_{\min }$ are maximum and minimum intensity, respectively.

Then, we design a triangle type membership function as shown in Figure 1. Thus, the membership degree $(\mu(I))$ is computed as following over the interval $\left[I_{\min }, I_{\max }\right]$.

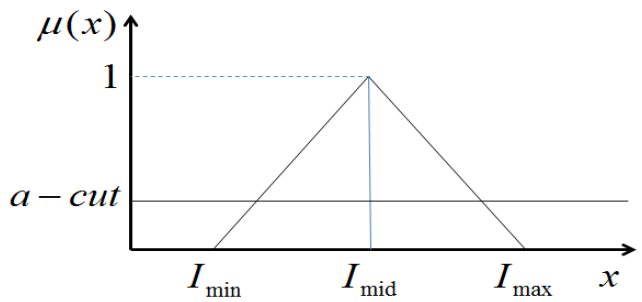

Figure 1. Fuzzy membership function for fuzzy brightness stretching

The cut point $(\alpha-c u t)$ in Figure 1 is computed as follows;

$$
\begin{aligned}
& \text { If }\left(I_{\text {min }}>0\right) \alpha-\text { cut }=I_{\text {min }} / I_{\text {max }}, \\
& \text { Else } \alpha-\text { cut }=0.5 \\
& X_{\text {new }}=255 \times \frac{X-\alpha}{\beta-\alpha},
\end{aligned}
$$

The upper limit value ( $\alpha$-cut) and the lower limit value $(\alpha)$ are defined as the highest and lowest $X_{i}$ among pixels that have higher membership degree than the cut point $\alpha$-cut.

The effect of such preprocessing is as shown in Figure 2.

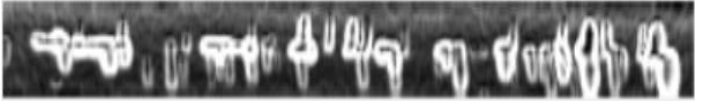

(a) Input image

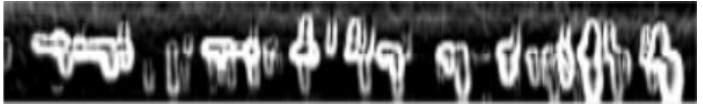

(b) After applying fuzzy stretching

Figure 2. The effect of fuzzy stretching

\subsection{Possibilistic C-Means pixel clustering with relaxation}

Then, the next step is applying pixel clustering algorithm to form the target object from the image obtained from above preprocessing step. In a standard FCM technique, a noisy pixel can be wrongly classified because of its abnormal feature data [30]. Possibilistic C-means (PCM) clustering is another unsupervised clustering method where the component generated by PCM corresponds to a dense region in the dataset. PCM is known to be more robust in the case of noisy data. The PCM membership degree refers to the degree of 'typicality' between data and clusters [26].

The PCM is based on the relaxation of the probabilistic constraint in order to interpret in a possibilistic sense of the membership function or degree of typicality.

Then we call $U_{p}=\left(u_{i j}\right)$ a possibilistic cluster partition of $X$ if :

$$
\sum_{j=1}^{N} u_{i j}>0, \forall i \in\{1, \ldots, \mathrm{c}\}
$$

The $u_{i j} \in[0,1]$ are interpreted as degree of typicality of the datum $\boldsymbol{x}_{j}$ to cluster $i$ and $u_{i j}$ for $\boldsymbol{x}_{\boldsymbol{j}}$ resembles the possibility of being a member of corresponding cluster.

The objective function in PCM clustering can be calculated as follows.

$$
J_{m}(L, U)=\sum_{i=1}^{C} \sum_{j=1}^{N}\left(u_{i j}\right)^{m} d_{i j}^{2}+\sum_{i=1}^{C} n_{i} \sum_{j=1}^{N}\left(1-u_{i j}^{m}\right)
$$


where $n_{i}$ denotes the average distance between points in the same group in this paper. The first term of equation (8) tries to minimize the distance between the data point and the cluster center and the second term is a penalty term to avoid having a trivial solution. The update formula for membership degree is defined as the (9).

$$
u_{i j}=\frac{1}{1+\left(\frac{d_{i j}}{n_{i}}\right)^{\frac{1}{m-1}}}
$$

The value $n_{i}$ decides the distance that the membership degree becomes 0.5 in the cluster. Thus, it represents the relative importance of the second term with respect to the first term in the objective function (equation (8)) and typically estimated as the (10) as following.

$$
n_{i}=K \times \frac{\sum_{j=1}^{N} u_{i j}^{m} d_{i j}^{2}}{\sum_{i=1}^{N} u_{i j}^{m}}
$$

where $K=1$ in this paper.

However, PCM has its own disadvantages such that it needs good initialization to obtain good result [23]. To classify a data point, cluster centroid has to be closest to the data point (by membership) and for estimating the centroids, the typicality needs to alleviate the undesirable effect of outliers. There exists a possibility that PCM stops with less number of clusters than the actual number of clusters.

Another problem arises in this standard PCM form in that the distance function as shown in (11) forces to converge to single winning cluster for the $\mathrm{j}^{\text {th }}$ data point $X_{j}$,

$$
d_{i j}=\left|X_{j}-V_{i}\right| \text { for } i \text { th cluster }
$$

In this paper, we relax this equation (11) as following in that the center value of $\mathrm{i}^{\text {th }}$ cluster is distributed to all clusters in a way to avoid local maxima where $V$ denotes the center value if there is only one cluster and there can be $c$ clusters.

$$
V_{i}=V+\sum_{i=0}^{c-1}(-1)^{i} \frac{V \times i}{2 c}
$$

The effect of such relaxation in this domain is as shown in Figure 3.

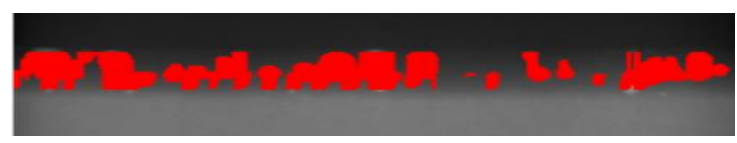

(a) Standard PCM

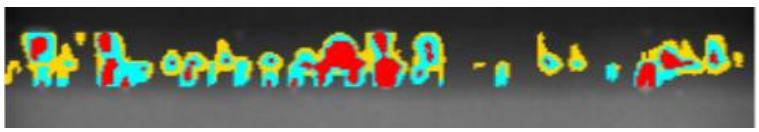

(b) Relaxed PCM

Figure 3. The effect of PCM

\section{EXPERIMENT AND RESULT}

The proposed method is implemented in C\# under Visual Studio 2017 environment with Intel(R) Dual Core(TM) i7-87005U CPU @ $2.0 \mathrm{GHz}$ and 32 GB RAM PC. Welding X-ray images are from Nondestructive Testing and Radiation Safety Center at Dong-Eui University, Pusan, Korea. Total 50 images for defect detection testing of ship building ceramic materials with various thicknesses $-8 \mathrm{~mm}, 10 \mathrm{~mm}, 11 \mathrm{~mm}$, $16 \mathrm{~mm}$, and $22 \mathrm{~mm}$. In order to compare the performance of proposed PCM clustering, we implement Kmeans clustering as used in [17]. The result is summarized in Table 1 and PCM proposed in this paper is far better than standard K-means clustering. One of the examples that the proposed method was successful but K-means was not for the same input is shown in Figure 4. Even when K-means seems to find the defect from the input, the proposed PCM gives richer result as shown in Figure 5. 
Table 1. Performance evaluation

\begin{tabular}{cccc}
\hline Thickness & K-Means & PCM & \#-of-Data \\
\hline $8 \mathrm{~mm}$ & 1 & 15 & 15 \\
$10 \mathrm{~mm}$ & 3 & 4 & 5 \\
$11 \mathrm{~mm}$ & 5 & 6 & 6 \\
$16 \mathrm{~mm}$ & 1 & 7 & 7 \\
$22 \mathrm{~mm}$ & 14 & 17 & 17 \\
Total & 24 & 49 & 50 \\
\hline
\end{tabular}

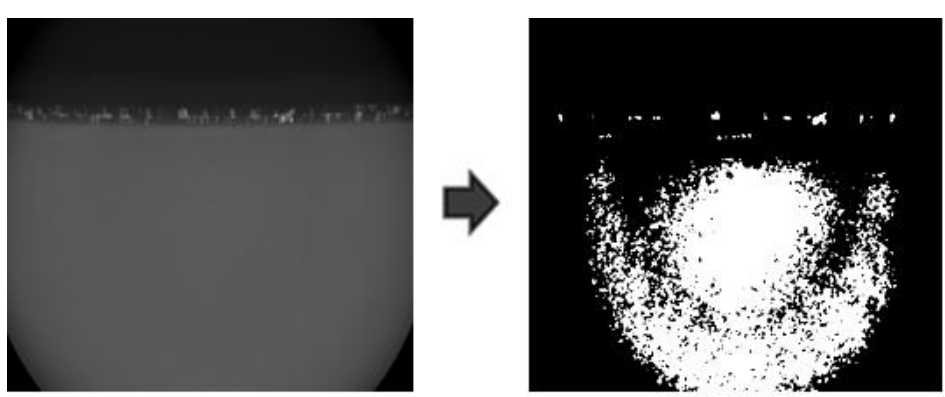

(a) K-means fails
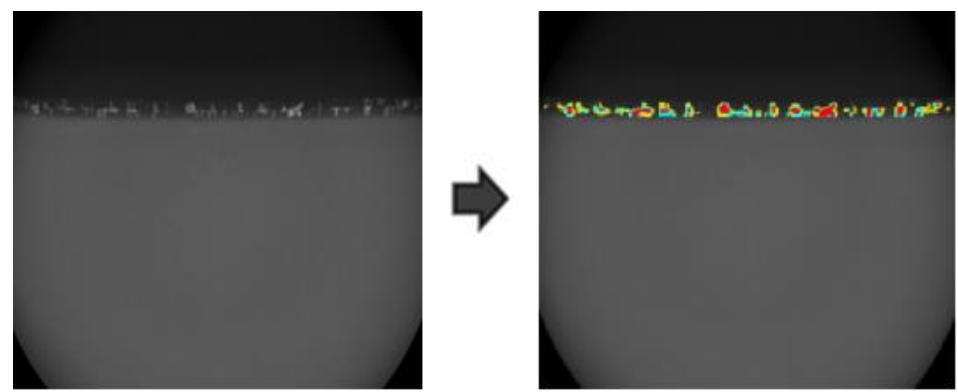

(b) Proposed PCM succeeds

Figure 4. Comparison of two methods - K-means failed case
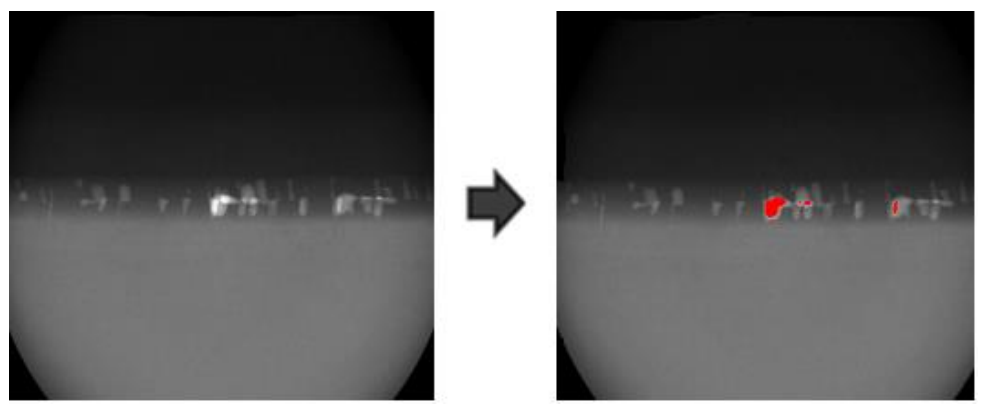

(a) K-means was successful
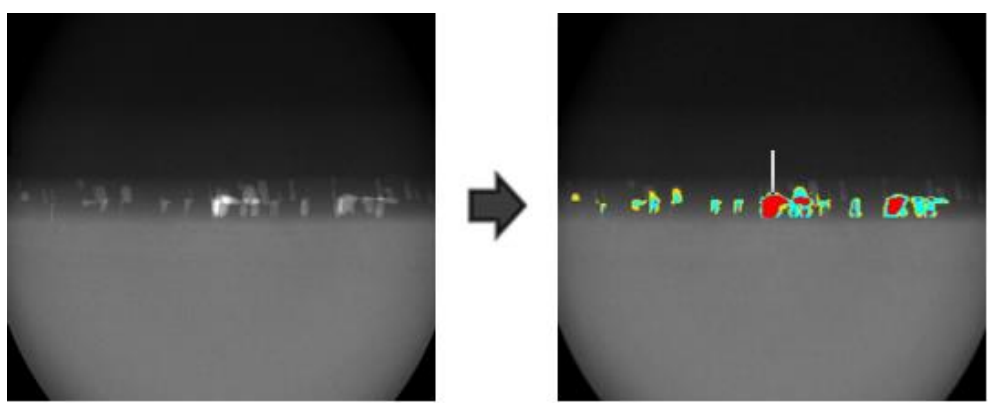

(b) PCM was successful and gives richer result

Figure 5. Comparison of two methods - K-means success case 


\section{CONCLUSION}

In this paper, we propose a method that extracts the suspicious defect from ceramic welding materials automatically through pixel clustering based segmentation. In preprocessing step, we apply standard Gaussian filtering and Sobel mask thus they are not the main reason of the obtained result. We apply an adaptive fuzzy clustering in image contrast enhancement and use PCM with relaxed cluster center belongings in distance computation. In experiment with 50 welding ceramic materials obtained from a university research center, the proposed PCM based method detects defects in 49 out of 50 cases. This result is far better than standard K-means clustering used as a competitor in this paper. The limitation of this paper is that the data used in the experiment is from only one center and the number of images are also limited thus the value of proposed method should be tested in the real world with more data.

\section{REFERENCES}

[1] K. B. Kim and W. Y Woo, "Detection of Flaws in Ceramic Materials Using Non-Destructive Testing," Journal of The Korea Institute of Electronic Communication Sciences, vol. 5, no. 3, pp. 321-326, 2010.

[2] K. B. Kim and D. H. Song, "Defect detection method using fuzzy stretching and ART2 learning from ceramic images," International Journal of Software Engineering and Its Applications, vol. 8, no. 9, pp. 29-38, 2014.

[3] A. Kumar, "Computer-vision-based fabric defect detection: a survey," Industrial Electronics, IEEE Transactions on 2008, vol. 55, no. 1, pp. 348-363, 2008.

[4] G. A. Ruz and P. A. Estévez, Image segmentation using fuzzy min-max neural networks for wood. In Proceedings of Intelligent Production Machines and Systems-First I* PROMS Virtual Conference: Elsevier, 2005.

[5] M. Thiruganam, S. M. Anouncia, and S. Kantipudi, "Automatic defect detection and counting in radiographic weldment images," International Journal of Computer Applications, vol. 10, no. 2, pp. 1-5, 2010.

[6] Z. Hocenski, S. Vasilic, and V. Hocenski, "Improved Canny Edge Detector in Ceramic Tiles Defect Detection," In Proc. of IEEE Industrial Electronics, IECON 2006-32nd Annual Conference on. IEEE, pp. 3328-3331, 2006.

[7] B. Lin, S. Chen, X. Han, and X. Liang, "Automatic damage detection of engineering ceramics ground surface based on texture analysis," Transactions of Tianjin University, vol. 19, no. 4, pp. 267-271, 2013.

[8] M. S. Mansoory, H. Tajik, G. Mohamadi, and M. Pashna, "Edge Defect Detection in Ceramic Tile Based on Boundary Analysis Using Fuzzy Thresholding and Radon Transform," In Signal Processing and Information Technology, IEEE International Symposium on, pp. 58-62, 2008.

[9] J. Silveira, M. J. O. Ferreira, C. Santos, and T. Martins, "Computer vision techniques applied to the quality control of ceramic plates," In Proc. of IEEE International Conference on Industrial Technology (ICIT 2009), 2009.

[10] C. R. Sarin, M. Karthik, M. Anilesh, and P. Subramaniam, "Advanced Image Enhancement of Ultrasonic Scan Images For Intelligent Quality Inspection of Adhesively Bonded Joints in Ceramics," International Journal of Advanced Research in Computer Science and Software Engineering, vol. 2, no. 4, pp. 302-306, 2012.

[11] P. A. Lestari, H. Rachmat, M. R. Ibrahim, and D. S. E. Atmaja, "Design of Automation System for Ceramic Surface Quality Control Using Artificial Neural Network at Balai Besar Keramik," In 2018 International Conference on Industrial Enterprise and System Engineering (IcoIESE 2018), 2019.

[12] R. Mishra and D. Shukla, "An automated ceramic tiles defect detection and classification system based on artificial neural network," Int. J. Emerg. Technol. Adv. Eng, vol. 4, no. 3, pp. 229-233, 2014.

[13] K. B. Kim, D. H. Song, and W. J. Lee, "Flaw detection in ceramics using sigma fuzzy binarization and gaussian filtering method," International Journal of Multimedia and Ubiquitous Engineering, vol. 9, no. 1, pp. 403-414, 2014.

[14] K. B. Kim and D. H. Song, "An Advanced Ceramic Defect Detection with Anisotropic Filtering and Diagonal Binarization," International Information Institute (Tokyo). Information, vol. 19, no. 1, pp. 181-187, 2016.

[15] K. B. Kim and D. H. Song, "Automatic Defect Detection using Fuzzy Binarization and Brightness Contrast Stretching from Ceramic Images for Non-Destructive Testing," Journal of the Korea Institute of Information and Communication Engineering, vol. 21, no. 11, pp. 2121-2127, 2017.

[16] H. J. Lee, D. H. Song, and K. B. Kim, "Effective Computer-Assisted Automatic Cervical Vertebrae Extraction with Rehabilitative Ultrasound Imaging by using K-means Clustering," International Journal of Electrical and Computer Engineering (IJECE), vol. 6, no. 6, pp. 2810-2817, 2016.

[17] K. B. Kim, D. H. Song, and S. S. Yun, "Automatic segmentation of wrist bone fracture area by K-means pixel clustering from X-ray image," International Journal of Electrical and Computer Engineering (IJECE), vol. 9, no. 6 , pp. 5205-5210, 2019.

[18] S. Abdelaziz and S. Lu, "K-means algorithm with level set for brain tumor segmentation," Indonesian Journal of Electrical Engineering and Computer Science, vol. 15, no. 2, pp. 991-1000, 2019.

[19] K. B. Kim, D. H. Song, and S. S. Yun, "Automatic Extraction of Blood Flow Area in Brachial Artery for Suspicious Hypertension Patients from Color Doppler Sonography with Fuzzy C-Means Clustering," Journal of information and communication convergence engineering, pp. 258-263, 2018.

[20] M. Ramezanifard and B. S. Mousavi, "Digital image classification by optimised fuzzy system," Indonesian Journal of Electrical Engineering and Computer Science, vol. 14, no. 3, pp. 1196-1202, 2019.

[21] S. N. Rehman and M. A. Hussain, "Fuzzy C-Means Algorithm Based Satellite Image Segmentation," Indonesian Journal of Electrical Engineering and Computer Science, vol. 9, no. 2, pp. 332-334, 2018.

[22] R. Krishnapuram and J. M. Keller, "ibilistic approach to clustering," IEEE Trans. Fuzzy Syst., vol. 12, pp. 98-110, 1993. 
[23] N. R. Pal, K. Pal, J. M. Keller, and J. C. Bezdek, “A possibilistic fuzzy c-means clustering algorithm," IEEE transactions on fuzzy systems, pp. 517-530, 2005.

[24] A. Bhat, "Possibility fuzzy c-means clustering for expression invariant face recognition," Int. J. Cybern. Inf., vol. 3, no. 2, pp. 35-45, 2014.

[25] A. Suryadibrata and K. B. Kim, "Ganglion Cyst Region Extraction from Ultrasound Images Using Possibilistic C-Means Clustering Method," Journal of information and communication convergence engineering, vol. 15, no. 1, pp. 49-52, 2017.

[26] S. R. Kannan, R. Devi, S. Ramathilagam, and T. P. Hong, "Effective fuzzy possibilistic c-means: an analyzing cancer medical database," Soft Computing, vol. 21, no. 11, pp. 2835-2845, 2017.

[27] J. Aparajeeta, P. K. Nanda, and N. Das, "Modified possibilistic fuzzy C-means algorithms for segmentation of magnetic resonance image," Applied Soft Computing, vol. 41, pp. 104-119, 2016.

[28] J. Peeples, M. Cook, D. Suen, A. Zare, and J. Keller, "Comparison of possibilistic fuzzy local information C-means and possibilistic K-nearest neighbors for synthetic aperture sonar image segmentation," In Detection and Sensing of Mines, Explosive Objects, and Obscured Targets XXIV, vol. 11012, pp. 110120T, 2019.

[29] R. C. Gonzales and R. E. Woods, Digital Image processing (3td Ed.), Prentice Hall, NJ, USA, 2007.

[30] K. S. Chuang, H. L. Tzeng, S. Chen, J. Wu, and T. J. Chen, "Fuzzy c-means clustering with spatial information for image segmentation," Computerized medical imaging and graphics, vol. 30, no. 1, pp. 9-15, 2006.

\section{BIOGRAPHIES OF AUTHORS}
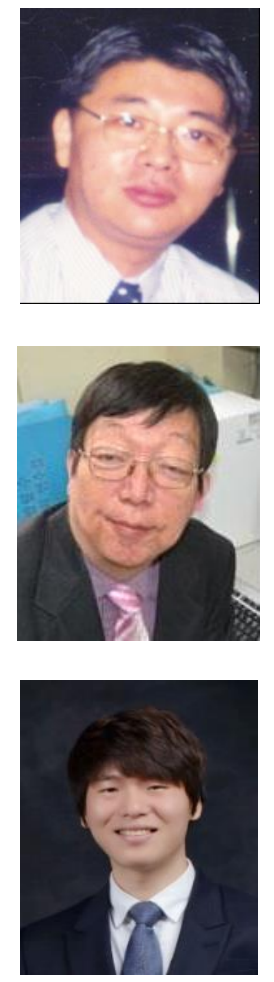

Kwang Baek Kim received his M.S. and Ph.D. degrees from the Department of Computer Science, Pusan National University, Busan, Korea, in 1993 and 1999, respectively. From 1997 to the present, he is a professor at the Division of Computer and Information Engineering, Silla University, Korea. He is currently an associate editor for Journal of Intelligence and Information Systems and The Journal of Information and Communication Convergence Engineering. His research interests include fuzzy clustering and fuzzy control system, computer vision, data mining, image processing, and bioinformatics.

Doo Heon Song received his B.S. from Seoul National University in 1981 and M.S. from the Korea Advanced Institute of Science and Technology in 1983 in Computer Science. He received his Ph.D. candidate Certificate in Computer Science from the University of California at Irvine in 1994. He has been a professor at Department of Computer Games, Yong-in Songdam College, Korea, since 1997. His research interests include machine learning, artificial intelligence, fuzzy systems, machine learning, and computer game design. He is currently the associate editor of Journal of Information and Comunication Convergence Engineeing.

Hyun Jun Park. He received his M.S. and Ph.D. degrees from the Department of Computer Engineering, Pusan National University, Busan, Korea, in 2009 and 2017, respectively. In 2017, he was a postdoctoral researcher at BK21PLUS, Creative Human Resource Development Program for IT Convergence, Pusan National University, Korea. From 2018 to the present, he is an associate professor at the Division of Software Convergence, Cheongju University, Korea. His research interests include computer vision, image processing, factory automation, neural network, and deep learning applications. 